\title{
cis-(-)-Menthyl phenylglycidates in the asymmetric synthesis of taxol side chain
}

\author{
Mustafa Er $^{1 *}$ and Necdet Coşkun ${ }^{2}$ \\ ${ }^{1}$ Muş Alparslan University, Department of Chemistry, 49100 Muş, Turkey \\ ${ }^{2}$ Uludăg University, Department of Chemistry, 16059 Görükle-Bursa, Turkey \\ E-mail: m.er@alparslan.edu.tr
}

\begin{abstract}
The one-pot azidation and benzoylation of a mixture of cis (-)-menthyl phenylglycidates provide quantitatively the corresponding (2R,3S)-, and (2S,3R)-3-azido-1-((1R,2S,5R)-2-isopropyl-5methylcyclohexyloxy)-1-oxo-3-phenylpropan-2-yl benzoate. Enantiopure (2R,3S)-3-azido-1((1R,2S,5R)-2-isopropyl-5-methylcyclohexyloxy)-1-oxo-3-phenylpropan-2-yl benzoate crystallize from $\mathrm{MeOH}$ at room temperature in high yields. The reduction of the latter with $\mathrm{Zn}-\mathrm{TMSCl}$ produces (-)-menthyl 3-benzamido-3-phenyl-2-(trimethylsilyloxy)propanoate which upon simultanious desilylation and hydrolysis provide the taxol side chain N-benzoyl-(2R,3S)-3phenylisoserine.
\end{abstract}

Keywords: $N$-Benzoyl-(2R,3S)-3-phenylisoserine, Taxol side chain, phenylglycidate, Zn-TMSCl reduction, azides

\section{Introduction}

The natural product Taxol, ${ }^{1}$ isolated from Taxus brevifolia, is considered the most promising anticancer drug. ${ }^{2}$ Because of the limited content in the bark of Taxus brevifolia, and the uneconomical production by total synthesis, ${ }^{3}$ extensive efforts have been focused on semisynthesis ${ }^{4}$ of taxol by the condensation of commercially available 10-deacetylbaccatin III with a side chain such as $N$-benzoyl-(2R,3S)-3-phenylisoserine (-)-5 (Figure 1). Therefore, the efficient synthesis of enantiopure side chain has attracted much attention from academic community as well as industry. ${ }^{5}$

Enantiomerically enriched phenylglycidates, are the most frequently used precursors of the taxol C-13 phenylisoserine side chain and diltiazem. ${ }^{\text {5a,e,f,i }}$ Asymmetric epoxidation and asymmetric dihydroxylation are the main methods used in the preparation of optically active cis and trans phenylglycidates. $^{6}$ An alternative way to prepare enantiopure epoxides is the asymmetric applications of the Darzen's reaction. ${ }^{7}$ 


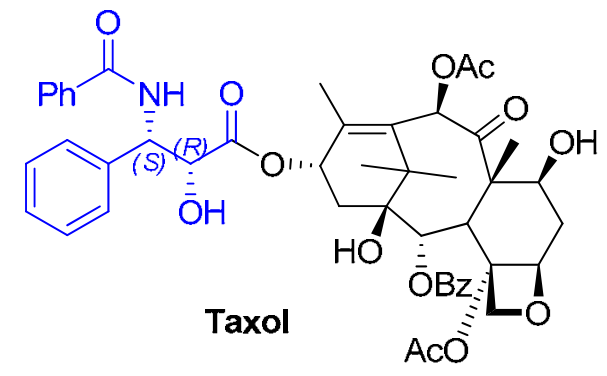

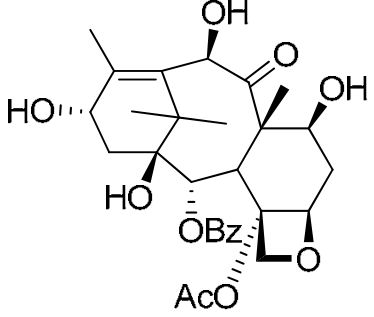

Baccatin III<smiles>O=C(N[C@H](O)C(=O)O)c1ccccc1</smiles>

$(-)-5$<smiles>O=C(N[C@H](C(=O)O)c1ccccc1)c1ccccc1</smiles>

$(+)-5$

Figure 1. Structure of taxol, baccatin III and taxol side chain enantiomers.

Here we report on the use of (2R,3R)- and (2S,3S)-(-)-menthyl 3-phenyloxirane-2-carboxylates ${ }^{8}$ 2-2' (Scheme 2) in the asymmetric syntheses of (2R,3S)- (-)-5 and (2S,3R)-3-benzoylamino-2hydroxy-3-phenylpropionic acid (+)-5 (Scheme 2).

\section{Results and Discussions}

Our retrosynthetic plan for the synthesis of taxol side chain is depicted in Scheme 1. Taxol side chain 5 can be prepared from the hydrolysis of menthyl ester 4 which in turn could be prepared by the reduction of azide 3 . One-pot reaction of menthyl glycidate 2 involving azidation and benzoylation will provide azide 3 . The mixture (65/35) of phenylglycidates $\mathbf{2}$ and $\mathbf{2}$ ' were converted to 3 and 3 ' in one-pot by direct treatment with $\mathrm{NaN}_{3}$ and following benzoylation (Sheme 2). Compound $\mathbf{3}$ crystallize in high yield from methanol at room temperature.

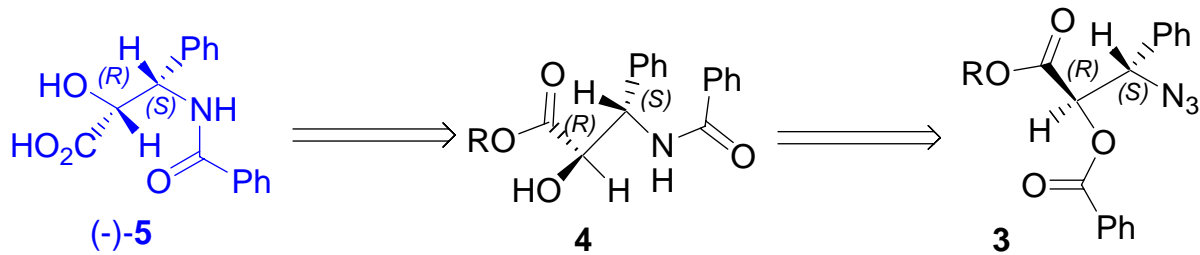

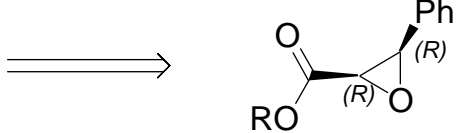

2<smiles>C#CC=C=C</smiles>

1

Scheme 1. Retrosynthetic analysis of the taxol side chain. 1-4; $R=(-)$-menthyl. 

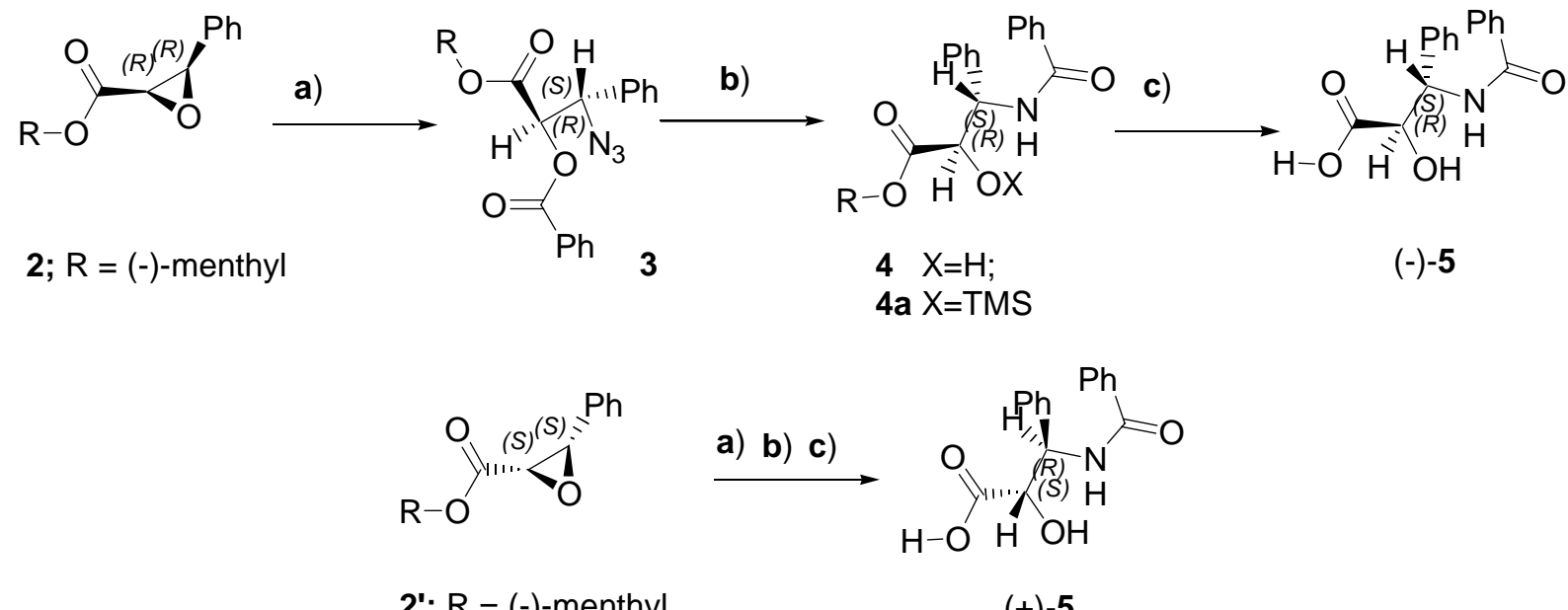

2'; R = (-)-menthyl (+)-5

Scheme 2. Reagents and conditions: (a) i) $\mathrm{NaN}_{3}$, DMF-ethylformate, $60{ }^{\circ} \mathrm{C}, 90 \mathrm{~h}$ ii) DMAP, benzoyl chloride, $\mathrm{CH}_{2} \mathrm{Cl}_{2}$, rt; 2 h, lit ${ }^{5 \mathrm{i}}$ (b) 5eq $\mathrm{Zn}-\mathrm{TMSCl}$, THF, reflux, $24 \mathrm{~h}$ (c) $\mathrm{KOH}$, TBAHS, THF, rt, $24 \mathrm{~h}$.

Compound 3 was treated with 5 eq. $\mathrm{Zn}$ and $\mathrm{Me}_{3} \mathrm{SiCl}$ heating at reflux in $\mathrm{THF}$ for $24 \mathrm{~h}$ to give the mixtures of (-)-menthyl 3-benzamido-2-hydroxy-3-phenylpropanoates 4 and menthyl 3-benzamido3-phenyl-2-(trimethylsilyloxy)propanoates 4a. The treatment of 4,4a with $\mathrm{KOH}$ in THF in the presence of a phase transfer catalyst gave the corresponding pure enantiomer $(2 \mathrm{R}, 3 \mathrm{~S})$-3-benzamido2-hydroxy-3-phenylpropanoic acid (-)-5. At similar conditions compound 2' (Scheme 2) was converted in one-pot into the taxol side chain enantiomer (2S,3R)-3-benzamido-2-hydroxy-3phenylpropanoic acid (+)-5.

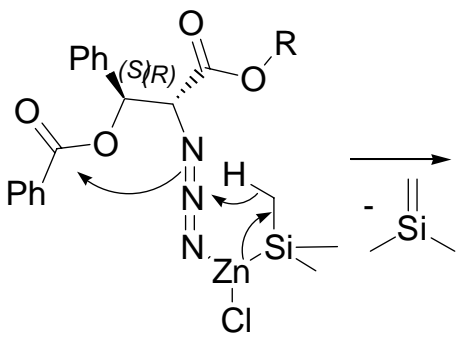

A

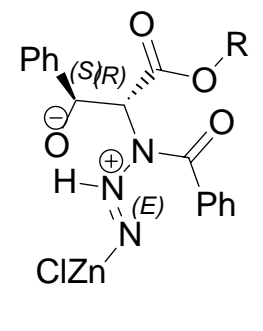

B
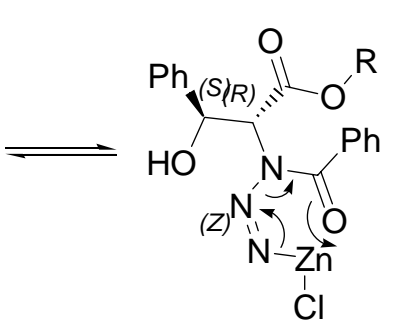

C

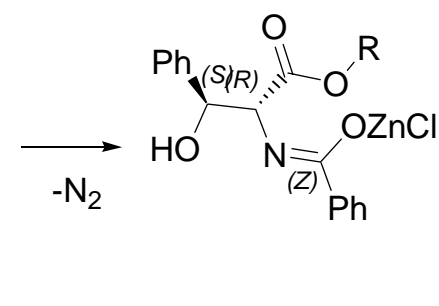

D

Scheme 3. Probable mechanism for the reduction of azides 3 with Zn-TMSCl.

The probable mechanism for the conversion of azide $\mathbf{3}$ to $\mathbf{4}$ is depicted in Scheme 3. We assume that single electron transfer from $\mathrm{Zn}$ to the $\mathrm{Si}$ in $\mathrm{TMSCl}$ can give $\mathrm{ClZnSiMe}_{3}$. The coordination of azide 3 to the metal centre of the latter through the terminal nitrogen would give intermediate $\mathbf{A}$ which synchronously abstracts dimethyl(methylene)silane to produce $\mathbf{B}$. The later is probably in equilibrium with $\mathbf{C}$ which eliminates $\mathrm{N}_{2}$ to give $\mathbf{D}$. The silylation of the latter give $4 \mathbf{a}$ or its hydrolysis produce the minor nonsilylated 4 . 


\section{Conclusions}

Thus, the products from the cis diastereoselective asymmetric Darzen condensation of benzaldehyde with (-)-menthyl haloacetate were demonstrated to be useful in the syntheses of the taxol side chain enantiomers. For the first time Zn-TMSCl system was employed in the reduction of the azide ${ }^{9}$ functionality of compounds 3-3' to give the corresponding silylated 4-4'. The latter were hydrolysed with $\mathrm{KOH}$ in the presence of a phase transfer catalyst such as tetrabutylammonium hydrogensulphate (TBAHS) to give the corresponding enantiopure taxol side chain enantiomers in high overall yields.

\section{Experimental Section}

General. Melting points were taken on an Electrothermal Digital melting point apparatus. Infrared spectra were recorded on a Thermo-Nicolet 6700 FTIR. 1D and 2D NMR spectra were recorded on a Varian Mercury Plus $400 \mathrm{MHz}$ spectrometer. Elemental analyses were performed on a EuroEA 3000 CHNS analyser.

TBAHS (97\% pure) were purchased from Aldrich. DMAP ( $\geq 99 \%$ GC), were Merck quality products. The THF ( $99 \%$ GC) was Riedel-de Haën product.

\section{Synthesis of (2R,3S)-3-benzamido-2-hydroxy-3-phenylpropanoic acid (Taxol side chain) (-)-5} Synthesis of (2R,3S)-3-azido-1-((1R,2S,5R)-2-isopropyl-5-methylcyclohexyloxy)-1-oxo-3phenyl- propan-2-yl benzoate (3). To a solution of menthyl phenylglycidate 2/2' ( 1 mmol, 302 $\mathrm{mg})$ in $\mathrm{MeOH}(9 \mathrm{~mL}), \mathrm{H}_{2} \mathrm{O}(1 \mathrm{~mL})$ and ethylformate $(1.5 \mathrm{~mL})$ mixture $\mathrm{NaN}_{3}(10 \mathrm{mmol}, 650 \mathrm{mg})$ was added and the reaction mixture stirred at $60{ }^{\circ} \mathrm{C}$ for $90 \mathrm{~h} .{ }^{5 \mathrm{i}}$ The mixture was cooled to room temperature and ethyl acetate $(25 \mathrm{~mL})$ was added and extracted with water $(3 \mathrm{X} 10 \mathrm{~mL})$. The organic phase was separated and dried over $\mathrm{Na}_{2} \mathrm{SO}_{4}$. The solvent was evaporated and the residue dissolved in $\mathrm{CH}_{2} \mathrm{Cl}_{2}(10 \mathrm{~mL})$. DMAP $(1 \mathrm{mmol}, 122 \mathrm{mg})$ was added and the mixture cooled to $0{ }^{\circ} \mathrm{C}$ then benzoyl chloride (1.24 mmol, $174 \mathrm{mg}$ ) was added drop-wise and the reaction mixture stirred at room temperature for $2 \mathrm{~h}$.The mixture was washed with water $(3 \mathrm{X} 10 \mathrm{~mL})$ and the organic phase dried over anhydrous $\mathrm{Na}_{2} \mathrm{SO}_{4}$, filtered and the solvent evaporated to produce an oily residue. Yield, $434 \mathrm{mg}, 92 \%$. The latter product was dissolved in methanol $(20 \mathrm{~mL})$ and left to crystallize in a refrigerator. The crystalline product was filtered and dried under vacuum to yield $368 \mathrm{mg}$, mixture of $\mathbf{3} / \mathbf{3}^{\prime}$ (The ratio determined by ${ }^{1} \mathrm{H}$ NMR is $65 / 35$ ). The latter mixture (368 $\mathrm{mg}$ ) was dissolved in $\mathrm{MeOH}(35 \mathrm{~mL})$ and left to crystallize overnight at room temperature. The formed crystalline 3 was filtered and dried under vacuum. White needles, mp 134-135 ${ }^{\circ} \mathrm{C}$; Yield $220 \mathrm{mg}, 49 \%$. $[\alpha]^{23}{ }_{\mathrm{D}}=+102$ (c, $\left.1.8, \mathrm{CHCl}_{3}\right)$. FTIR (KBr); $v_{\mathrm{N} 3} 2103, v_{\mathrm{C}=\mathrm{O}} 1737,1723 \mathrm{~cm}^{-1} .{ }^{1} \mathrm{H}$ NMR $\left(400 \mathrm{MHz}, \mathrm{CDCl}_{3}\right) \delta \mathrm{ppm}$ $0.63(3 \mathrm{H}, \mathrm{d}, J=6.8 \mathrm{~Hz}), 0.73(3 \mathrm{H}, \mathrm{d}, J=6.8 \mathrm{~Hz}), 0.79-0.88(1 \mathrm{H}, \mathrm{m}), 0.90(3 \mathrm{H}, \mathrm{d}, J=6.4 \mathrm{~Hz}), 0.94-$ $1.07(2 \mathrm{H}, \mathrm{m}), 1.28-1.35(1 \mathrm{H}, \mathrm{m}), 1.40-1.53(2 \mathrm{H}, \mathrm{m}) 1.59-1.67(2 \mathrm{H}, \mathrm{m}) 2.02-2.07(1 \mathrm{H}, \mathrm{m}), 4.69(1 \mathrm{H}$, dt, $J=10.8,4.4 \mathrm{~Hz}), 5.15(1 \mathrm{H}, \mathrm{d}, J=5.2 \mathrm{~Hz}), 5.45(1 \mathrm{H}, \mathrm{d}, J=5.2 \mathrm{~Hz}), 7.35-7.41(3 \mathrm{H}, \mathrm{m}), 7.43-$ $7.49(4 \mathrm{H}, \mathrm{m}), 7.57-7.61(1 \mathrm{H}, \mathrm{m}), 8.07-8.09(2 \mathrm{H}, \mathrm{m}) .{ }^{13} \mathrm{C} \mathrm{NMR}\left(100 \mathrm{MHz}, \mathrm{CDCl}_{3}\right) \delta \mathrm{ppm} \mathrm{15.6}$; 
$20.8 ; 21.9 ; 22.8 ; 25.5 ; 31.4 ; 34.1 ; 40.4 ; 46.6 ; 65.6 ; 75.6 ; 76.6 ; 127.7 ; 128.5 ; 128.9 ; 129.0 ; 129.1$; 130.0; 133.6; 134.6; 165.6; 166.9. Anal Calc for $\mathrm{C}_{26} \mathrm{H}_{31} \mathrm{~N}_{3} \mathrm{O}_{4}$ (449.23) C, 69.47; H, 6.95; N, 9.35; Found C, 69.46; H, 6.97; N, 9.38.

(2S,3R)-3-azido-1-((1R,2S,5R)-2-isopropyl-5-methylcyclohexyloxy)-1-oxo-3-phenylpropan-2-yl benzoate 3'. FTIR $\left(\mathrm{v} \mathrm{cm}^{-1}\right): 2103,1738,1723{ }^{1} \mathrm{H}$ NMR $\left(400 \mathrm{MHz}, \mathrm{CDCl}_{3}\right)$ (The spectrum is elicited from the spectrum of a mixture containing $90 \% 3^{\prime}$.) $\delta \mathrm{ppm} 0.76(3 \mathrm{H}, \mathrm{d}, J=7.2), 0.84(3 \mathrm{H}$, d, $J=6.8), 0.88(3 \mathrm{H}, \mathrm{d}, J=6.4), 0.80-0.88(1 \mathrm{H}, \mathrm{m}), 0.94-1.08(2 \mathrm{H}, \mathrm{m}), 1.27-1.36(1 \mathrm{H}, \mathrm{m}), 1.41-$ $1.54(2 \mathrm{H}, \mathrm{m}) 1.60-1.68(2 \mathrm{H}, \mathrm{m}) 2.01-2.07(1 \mathrm{H}, \mathrm{m}), 4.70(1 \mathrm{H}, \mathrm{dt}, J=10.8,4.4), 5.13(1 \mathrm{H}, \mathrm{d}, J=$ 5.2), $5.47(1 \mathrm{H}, \mathrm{d}, J=5.2), 7.34-7.41(3 \mathrm{H}, \mathrm{m}), 7.43-7.49(4 \mathrm{H}, \mathrm{m}), 7.58-7.62(1 \mathrm{H}, \mathrm{m}), 8.07-8.10(2 \mathrm{H}$, m). ${ }^{13} \mathrm{C}$ NMR $\left(100 \mathrm{MHz}, \mathrm{CDCl}_{3}\right) \delta \mathrm{ppm} 16.2 ; 20.7 ; 21.9 ; 23.2 ; 26.1 ; 31.3 ; 34.0 ; 40.0 ; 46.8 ; 65.7$; $75.5 ; 76.3 ; 127.8 ; 128.5 ; 128.9 ; 129.0 ; 129.1 ; 130.0 ; 133.5 ; 134.7 ; 165.5 ; 167.0$

Anal Calc for $\mathrm{C}_{26} \mathrm{H}_{31} \mathrm{~N}_{3} \mathrm{O}_{4}(449.23)$ C, 69.47; H, 6.95; N, 9.35; Found C, 69.42; H, 6.92; N, 9.42.

\section{Reduction of compound 3. General procedure}

The mixture of zinc powder ( $5 \mathrm{mmol}, 325 \mathrm{mg}$ ) and $\mathrm{Me}_{3} \mathrm{SiCl}(5 \mathrm{mmol}, 540 \mathrm{mg})$ in $\mathrm{THF}(10 \mathrm{~mL})$ was stirred for 5 minutes at room temperature. Benzoylated azide $3(1 \mathrm{mmol})$ was added to the mixture and heated at reflux for $24 \mathrm{~h}$. The unreacted zinc powder was filtered and water $(10 \mathrm{~mL})$ was added to the cooled mixture and extracted with ethyl acetate $(3 \mathrm{X} 10 \mathrm{~mL})$. The organic phase was dried over anhydrous $\mathrm{Na}_{2} \mathrm{SO}_{4}$ filtered and the solvent evaporated. The residue was subjected to silica packed column and eluted with ethyl acetate and petroleum ether.

(2R,3S)-((1R,2S,5R)-2-Isopropyl-5-methylcyclohexyl) 3-benzamido-2-hydroxy-3-phenylpropanoate (4). Oil. Yield $102 \mathrm{mg}, 24 \%$. $[\alpha]_{\mathrm{D}}^{22}=-78\left(\mathrm{c}, 0.5, \mathrm{CHCl}_{3}\right.$ ). FTIR (neat): $v_{\mathrm{OH}} 3520, v_{\mathrm{NH}} 3345, v_{\mathrm{C}=\mathrm{O}} 1725$, $1645 \mathrm{~cm}^{-1} .{ }^{1} \mathrm{H}$ NMR $\left(400 \mathrm{MHz}, \mathrm{CDCl}_{3}\right) \delta \mathrm{ppm} 0.52(3 \mathrm{H}, \mathrm{d}, J=7.2 \mathrm{~Hz}), 0.76(3 \mathrm{H}, \mathrm{d}, J=7.2 \mathrm{~Hz})$, $0.91(3 \mathrm{H}, \mathrm{d}, J=6.4 \mathrm{~Hz}), 0.82-0.92(1 \mathrm{H}, \mathrm{m}), 0.94-1.10(2 \mathrm{H}, \mathrm{m}), 1.39-1.50(2 \mathrm{H}, \mathrm{m}), 1.63-1.71(2 \mathrm{H}$, m), 1.76-1.83 $(1 \mathrm{H}, \mathrm{m}), 1.94-1.99(1 \mathrm{H}, \mathrm{m}), 3.33(1 \mathrm{H}, \mathrm{d}, J=3.2 \mathrm{~Hz}, \mathrm{C} 2-\mathrm{OH}), 4.57(1 \mathrm{H}, \mathrm{dd}, J=3.2$, $2.0 \mathrm{~Hz}, \mathrm{C} 2-\mathrm{H}), 4.84(1 \mathrm{H}, \mathrm{dt}, J=10.8,4.4 \mathrm{~Hz}, \mathrm{C} 1-\mathrm{H}), 5.69(1 \mathrm{H}, \mathrm{dd}, J=8.98,2.0 \mathrm{~Hz}, \mathrm{C} 3-\mathrm{H}), 7.11$ $(1 \mathrm{H}, \mathrm{d}, J=8.98 \mathrm{~Hz}, \mathrm{NH}), 7.27-7.31(1 \mathrm{H}, \mathrm{m}), 7.33-7.37(2 \mathrm{H}, \mathrm{m}), 7.42-7.46(4 \mathrm{H}, \mathrm{m}), 7.49-7.53(1 \mathrm{H}$, m), 7.76-7.79 (2H, m). ${ }^{13} \mathrm{C}$ NMR $\left(100 \mathrm{MHz}, \mathrm{CDCl}_{3}\right) \delta \mathrm{ppm} 15.4 ; 20.8 ; 21.9 ; 22.7 ; 25.6 ; 29.7 ; 31.5$; $34.0 ; 40.7 ; 46.7 ; 54.7 ; 73.8 ; 126.8 ; 127.1 ; 127.8 ; 128.5 ; 128.6 ; 131.7 ; 133.9 ; 138.9 ; 166.4 ; 172.5$. Anal Calc for $\mathrm{C}_{26} \mathrm{H}_{33} \mathrm{NO}_{4}$ (423.24) C, 73.73; H, 7.85; N, 3.31; Found C, 73.78; H, 7.81; N, 3.35. (2R,3S)-((1R,2S,5R)-2-isopropyl-5-methylcyclohexyl) 3-benzamido-3-phenyl-2-(trimethylsilyloxy) propanoate (4a). Oil. Yield $287 \mathrm{mg}, 58 \%$. [ $\alpha]_{\mathrm{D}}^{22}=-76\left(\mathrm{c}, 0.3, \mathrm{CHCl}_{3}\right)$. FTIR $(\mathrm{KBr}): \mathrm{NH}_{3} 346, v_{\mathrm{C}=\mathrm{O}}$ $1728,1645 \mathrm{~cm}^{-1} .{ }^{1} \mathrm{H}$ NMR $\left(400 \mathrm{MHz}, \mathrm{CDCl}_{3}\right) \delta \mathrm{ppm}-0.13(9 \mathrm{H}, \mathrm{s}, \mathrm{TMS}), 0.57(3 \mathrm{H}, \mathrm{d}, J=7.2 \mathrm{~Hz})$, $0.80(3 \mathrm{H}, \mathrm{d}, J=7.2 \mathrm{~Hz}), 0.87(3 \mathrm{H}, \mathrm{d}, J=6.8 \mathrm{~Hz}), 0.84-0.90(1 \mathrm{H}, \mathrm{m}), 0.95-1.04(2 \mathrm{H}, \mathrm{m}), 1.38-1.48$ $(2 \mathrm{H}, \mathrm{m}), 1.63-1.68(2 \mathrm{H}, \mathrm{m}), 1.79-1.93(2 \mathrm{H}, \mathrm{m}), 4.41(1 \mathrm{H}, \mathrm{d}, J=1.56 \mathrm{~Hz}, \mathrm{C} 2-\mathrm{H}), 4.80(1 \mathrm{H}, \mathrm{dt}, J=$ 10.8, 4.4 Hz, C1-H), $5.57(1 \mathrm{H}, \mathrm{dd}, J=8.19,1.56 \mathrm{~Hz}, \mathrm{C} 3-\mathrm{H}), 7.20(1 \mathrm{H}, \mathrm{d}, J=8.19 \mathrm{~Hz}, \mathrm{NH}), 7.23-$ 7.28 $(1 \mathrm{H}, \mathrm{m}), 7.30-7.38(4 \mathrm{H}, \mathrm{m}), 7.42-7.46(2 \mathrm{H}, \mathrm{m}), 7.49-7.53(1 \mathrm{H}, \mathrm{m}), 7.80-7.82(2 \mathrm{H}, \mathrm{m}) .{ }^{13} \mathrm{C}$ NMR $\left(100 \mathrm{MHz}, \mathrm{CDCl}_{3}\right) \delta \mathrm{ppm}-0.7 ; 15.6 ; 20.9 ; 22.0 ; 22.8 ; 25.7 ; 31.4 ; 34.1 ; 40.9 ; 46.9 ; 56.3 ; 75.3$; $75.6 ; 126.7 ; 127.2 ; 127.5 ; 128.3 ; 128.5 ; 131.6 ; 134.3 ; 139.4 ; 166.4 ; 170.9$. Anal Calc for $\mathrm{C}_{29} \mathrm{H}_{41} \mathrm{NO}_{4} \mathrm{Si}(495.28) \mathrm{C}, 70.26 ; \mathrm{H}, 8.34 ; \mathrm{N}, 2.83$; Found C, 70.35; H, 8.38; N, 2.91. 


\section{(2R,3S)-3-Benzamido-2-hydroxy-3-phenylpropanoic acid (Taxol side chain) (-)-5. General procedure}

To a solution of 4 or $\mathbf{4 a}(1 \mathrm{mmol})$ in THF $(10 \mathrm{~mL})$ TBAHS $(0.1 \mathrm{mmol}, 33.9 \mathrm{mg})$ and $\mathrm{KOH}(1$ mmol, $56 \mathrm{mg}$ ) were added successively and the reaction mixture stirred at room temperature for 24 h. Water was added $(15 \mathrm{~mL})$ to the mixture and extracted with ethyl acetate $(3 \mathrm{X} 10 \mathrm{~mL})$. The water phase was acidified with $1 \mathrm{~N} \mathrm{HCl}$ to $\mathrm{pH} 2$ and the precipitated solid filtered. From 4, yield $193 \mathrm{mg}$, $68 \%$. Mp 176-178 ${ }^{\circ} \mathrm{C} ;[\alpha]^{22}{ }_{\mathrm{D}}=-33.3(\mathrm{c}, 0.32, \mathrm{EtOH}) . \mathrm{Lit}^{10} \mathrm{mp} 175.5-177.8^{\circ} \mathrm{C}$ and $[a]_{\mathrm{D}}{ }^{22}=-35.5(\mathrm{c}$ 1.07, EtOH). From 4a, yield 0.185, 65\%, $[\alpha]_{\mathrm{D}}^{22}=-33.3$ (c, 0.32, EtOH). Mp 176-178 ${ }^{\circ} \mathrm{C}$. FTIR $(\mathrm{KBr}) ; v_{\mathrm{OH}} 3523, v_{\mathrm{NH}} 3350, v_{\mathrm{C}=\mathrm{O}} 1707,1641 \mathrm{~cm}^{-1} .{ }^{1} \mathrm{H}$ NMR $(400 \mathrm{MHz}, \mathrm{DMSO}-\mathrm{d} 6) \delta \mathrm{ppm} 3.31(2 \mathrm{H}$, brs), $4.36(1 \mathrm{H}, \mathrm{d}, J=4.3 \mathrm{~Hz}), 5.45(1 \mathrm{H}, \mathrm{dd}, J=9.0,4.3 \mathrm{~Hz}), 7.21-7.24(1 \mathrm{H}, \mathrm{m}), 7.30(2 \mathrm{H}, \mathrm{t}, J=7.2$ $\mathrm{Hz}), 7.38-7.39(2 \mathrm{H}, \mathrm{m}), 7.46-7.54(3 \mathrm{H}, \mathrm{m}), 7.81-7.84(2 \mathrm{H}, \mathrm{m}), 8.56(1 \mathrm{H}, \mathrm{d}, J=9.0 \mathrm{~Hz}) .{ }^{13} \mathrm{C}$ NMR $\left(100 \mathrm{MHz}, \mathrm{CDCl}_{3}\right) \delta$ ppm 56.3; 74.0; 127.4; 127.6; 127.8; 128.5; 128.8; 131.8; 134.8; 140.7; 166.5; 173.9 Anal Calc for $\mathrm{C}_{16} \mathrm{H}_{15} \mathrm{NO}_{4}(285.10) \mathrm{C}, 67.36 ; \mathrm{H}, 5.30 ; \mathrm{N}, 4.91$; Found C, 67.38; H, 5.32; N, 4.98 .

Synthesis of (2R,3S)-3-azido-2-hydroxy-3-phenylpropionic acid (1R,2S,5R)-2-isopropyl-5methyl-cyclohexyl ester

To a solution of (-)-menthyl phenylglycidate 2 (1 mmol, $302 \mathrm{mg})$ in $\mathrm{MeOH}(9 \mathrm{~mL}), \mathrm{H}_{2} \mathrm{O}(1 \mathrm{~mL})$ and ethylformate $(1.5 \mathrm{~mL})$ mixture $\mathrm{NaN}_{3}(10 \mathrm{mmol}, 650 \mathrm{mg})$ was added and the reaction mixture stirred at $60{ }^{\circ} \mathrm{C}$ for $90 \mathrm{~h}$. The mixture was cooled to room temperature and ethyl acetate $(25 \mathrm{~mL})$ was added and extracted with water $(3 \mathrm{X} 10 \mathrm{~mL})$. The organic phase was separated and dried over

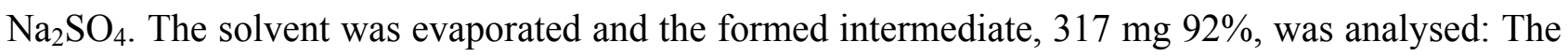
purity was controlled by ${ }^{1} \mathrm{H}$ NMR and shown to be $100 \%$. FTIR (KBr); $v_{\mathrm{OH}} 3483, v_{\mathrm{N} 3} 2106, v_{\mathrm{C}=\mathrm{O}}$ $1731 \mathrm{~cm}^{-1} .{ }^{1} \mathrm{H}$ NMR $\left(400 \mathrm{MHz}, \mathrm{CDCl}_{3}\right) \delta \mathrm{ppm} 0.79(3 \mathrm{H}, \mathrm{d}, J=6.8 \mathrm{~Hz}), 0.93(6 \mathrm{H}, \mathrm{d}, J=6.8 \mathrm{~Hz})$, 0.87-0.91 (1H, m), 0.97-1.13 (2H, m), 1.42-1.54 (2H, m), 1.69-1.74 (2H, m), 1.79-1.86 (1H, m), 2.02-2.07 (1H, m), $3.12(1 \mathrm{H}, \mathrm{d}, J=6.8 \mathrm{~Hz}, \mathrm{C} 2-\mathrm{OH}), 4.33(1 \mathrm{H}, \mathrm{dd}, J=6.8,2.8 \mathrm{~Hz}, \mathrm{C} 2-\mathrm{H}), 4.86(1 \mathrm{H}$, d, $J=2.8 \mathrm{~Hz}, \mathrm{C} 3-\mathrm{H}), 4.84(1 \mathrm{H}, \mathrm{dt}, J=10.8,4.4 \mathrm{~Hz}, \mathrm{C} 1-\mathrm{H}), 7.35-7.44(3 \mathrm{H}, \mathrm{m}), 7.47-7.50(2 \mathrm{H}, \mathrm{m})$. ${ }^{13} \mathrm{C}$ NMR $\left(100 \mathrm{MHz}, \mathrm{CDCl}_{3}\right) \delta$ ppm $15.6 ; 20.9 ; 22.0 ; 22.9 ; 26.1 ; 31.4 ; 34.1 ; 40.7 ; 46.9 ; 67.1 ; 73.8$; 77.1; 128.0; 128.7; 128.8; 135.6; 171.6. Anal Calc for $\mathrm{C}_{19} \mathrm{H}_{27} \mathrm{~N}_{3} \mathrm{O}_{3}(345.21) \mathrm{C}, 66.06 ; \mathrm{H}, 7.88 ; \mathrm{N}$, 12.16; Found C, 66.08; H, 7.92; N, 12.20.

The product obtained from the benzoylation of the latter was identical with those of $\mathbf{3}$.

\section{One-pot procedure for the synthesis of (2S,3R)-3-benzamido-2-hydroxy-3-phenylpropanoic acid $(+)-5$ (Taxol side chain enantiomer)}

Menthyl phenylglycidate 2' (3 mmol, $906 \mathrm{mg}$ ) was dissolved in a mixture containing $\mathrm{MeOH}(27$ $\mathrm{mL}), \mathrm{H}_{2} \mathrm{O}(3 \mathrm{~mL})$ and ethylformate $(5 \mathrm{~mL}) . \mathrm{NaN}_{3}(30 \mathrm{mmol}, 1950 \mathrm{mg})$ was added and the reaction mixture was stirred at $60{ }^{\circ} \mathrm{C}$ for $90 \mathrm{~h}$. The work up procedure is as described above. To a cooled to 0 ${ }^{\circ} \mathrm{C}$ solution of the nearly pure azidoalcohol $(2.72 \mathrm{mmol}, 940 \mathrm{mg})$ in $\mathrm{CH}_{2} \mathrm{Cl}_{2}(15 \mathrm{~mL})$ and DMAP (2.72 mmol, $332 \mathrm{mg})$, benzoyl chloride $(3.41 \mathrm{mmol}, 479 \mathrm{mg}$ ) was added drop-wise and the reaction mixture stirred at room temperature for $2 \mathrm{~h}$. The mixture was washed with water $(3 \mathrm{X} 15 \mathrm{~mL})$ and the organic phase dried over anhydrous $\mathrm{Na}_{2} \mathrm{SO}_{4}$, filtered and the oily residue, $1060 \mathrm{mg}$, was dissolved 
in methanol. The crystalline product was filtered and dried under vacuum to yield $950 \mathrm{mg} \mathbf{3}$ '. To the mixture of zinc powder (10.5 mmol, $687 \mathrm{mg}$ ) and $\mathrm{Me}_{3} \mathrm{SiCl}(10.5 \mathrm{mmol}, 1134 \mathrm{mg})$ in THF (20 mL) benzoylated azide 3' was added and the mixture refluxed for $24 \mathrm{~h}$. The unreacted zinc powder was filtered and water $(15 \mathrm{~mL})$ was added to the cooled mixture and extracted with ethyl acetate (3X15 $\mathrm{mL}$ ). The organic phase was dried over anhydrous $\mathrm{Na}_{2} \mathrm{SO}_{4}$ filtered and the solvent evaporated. To the residue containing the $\mathbf{4}^{\prime}$ and $\mathbf{4}^{\prime} \mathbf{a}$ (1000 $\mathrm{mg}, 68 / 32$ by ${ }^{1} \mathrm{H}$ NMR spectroscopy) dissolved in THF $(20 \mathrm{~mL})$ TBAHS $(0.21 \mathrm{mmol}, 71 \mathrm{mg})$ and $\mathrm{KOH}(2.68 \mathrm{mmol}, 156 \mathrm{mg})$ were added successively and the reaction mixture stirred at room temperature for $24 \mathrm{~h}$. Water was added $(15 \mathrm{~mL})$ to the mixture and extracted with ethyl acetate $(3 \mathrm{X} 10 \mathrm{~mL})$. The layers were separated and the water phase was acidified with $1 \mathrm{~N} \mathrm{HCl}$ to $\mathrm{pH} 2$ and the precipitated solid filtered to give $371 \mathrm{mg}, 69.5 \%(+)-5$. Mp $179-181^{\circ} \mathrm{C} .[\alpha]^{20}{ }_{\mathrm{D}}=+38.8$ (c, 0.73, EtOH). FTIR (KBr); $v_{\mathrm{OH}} 3523, v_{\mathrm{NH}} 3350, v_{\mathrm{C}=0} 1707,1641 \mathrm{~cm}^{-1}$. ${ }^{1} \mathrm{H}$ NMR (400 MHz, DMSO-d6) $\delta$ ppm $3.31(2 \mathrm{H}, \mathrm{brs}), 4.36(1 \mathrm{H}, \mathrm{d}, J=4.3 \mathrm{~Hz}), 5.45(1 \mathrm{H}, \mathrm{dd}, J=$ 9.0; 4.3 Hz) , 7.21-7.24 (1H, m), 7.30 (2H, t, $J=7.2 \mathrm{~Hz}), 7.38-7.39(2 \mathrm{H}, \mathrm{m}), 7.46-7.54(3 \mathrm{H}, \mathrm{m})$, 7.81-7.84 $(2 \mathrm{H}, \mathrm{m}), 8.56(1 \mathrm{H}, \mathrm{d}, J=9.0 \mathrm{~Hz}) .{ }^{13} \mathrm{C} \mathrm{NMR}\left(100 \mathrm{MHz}, \mathrm{CDCl}_{3}\right) \delta \mathrm{ppm} 56.3 ; 74.0 ; 127.4$; $127.6 ; 127.8 ; 128.5 ; 128.8 ; 131.8 ; 134.8 ; 140.7 ; 166.5 ; 173.9$ Anal Calc for $\mathrm{C}_{16} \mathrm{H}_{15} \mathrm{NO}_{4}(285.10) \mathrm{C}$, 67.36; H, 5.30; N, 4.91; Found C, 67.40; H, 5.35; N, 4.93.

\section{Acknowledgements}

Uludă̆ University Research Fund is gratefully acknowledged for its financial support (Project No 2001-2).

\section{References}

1. (a) Wani, M. C.; Taylor, H. I.; Wall, M. E.; Coggon, P.; McPhail, A. T. J. Am. Chem. Soc. 1971, 93, 2325. (b) Kingston, D. G. I. Chem. Commun. 2001, 867. (c) Miller, M. L.; Ojima, I. Chem. Rec. 2001, 1, 195. (d) Horwitz, S. B. J. Nat. Prod. 2004, 67, 136.

2. (a) Mathew, A. E.; Mejillano, M. R.; Nath, J. P.; Himes, R. H.; Stella, V. J. J. Med. Chem. 1992, 35, 145. (b) Georg, G. I.; Cheruvallath, Z.S.; Himes, R.H.; Mejillano, M.R.; Burke, C.T. J. Med. Chem. 1992, 35, 4230.

3. (a) Kusama, H.; Hara, R.; Kawahara, S.; Nishimori, T.; Kashima, H.; Nakamura, N.; Morihira, K.; Kuwajima, I. J. Am. Chem. Soc. 2000, 122, 3811. (b) Mukaiyama, T.; Shiina, I.; Iwadare, H.; Saitoh, M.; Nishimura, T.; Ohkawa, N.; Sakoh, H.; Nishimura, K.; Tani, Y.; Hasegawa, M.; Yamada, K.; Saitoh, K. Chem.-Eur. J. 1999, 5, 121. (c) Wender, P. A.; Badham, N. F.; Conway, S. P.; Floreancig, P. E.; Glass, T. E.; Granicher, C.; Houze, J. B.; Janichen, J.; Lee, D.; Marquess, D. G.; McGrane, P. L.; Meng, W.; Mucciaro, T. P.; Muhlebach, M.; Natchus, M. G.; Paulsen, H.; Rawlins, D. B.; Satkofsky, J.; Shuker, A. J.; Sutton, J. C.; Tayler, R. E.; Tomooka, K. J. Am. Chem. Soc. 1997, 119, 2755. (d) Master, J. J.; Link, J. T.; Snyder, L. B.; Young, W. B.; Danishefsky, S. J. Angew. Chem., Int. Ed. 1995, 34, 1723. (e) Holton, R. A.; Somoza, C.; Kim, 
H.-B.; Liang, F.; Biediger, R. J.; Boatman, P. D.; Shindo, M.; Smith, C. C.; Kim, S.; Nadizadeh, H.; Suzuki, Y.; Tao, C.; Vu, P.; Tang, S.; Zhang, P.; Murthi, K. K.; Gentile, L. N.; Liu, J. H. J. Am. Chem. Soc. 1994, 116, 1597. (f) Nicolaou, K. C.; Yang, Z.; Liu, J. J.; Ueno, H.; Nantermet, P. G.; Guy, R. K.; Claiborne, C. F.; Renaud, J.; Couladouros, E. A.; Paulvannan, K.; Sorensen, E. J. Nature 1994, 367, 630.

4. (a) Gennari, C.; Vulpetti, A.; Donghi, M.; Mongelli, N.; Vanotti, E. Angew. Chem., Int. Ed. 1996, 35, 1723. (b) Ojima, I. Acc. Chem. Res. 1995, 28, 383. (c) Ojima, I.; Habus, I.; Zhao, M.; Zucco, M.; Park, Y. H. Tetrahedron 1992, 48, 6985.

5. (a) Anand, N.; Kapoor, M.; Koul, S.; Taneja, S.C.; Sharmab, R.L.; Qazia, G.N. Tetrahedron: Asymm. 2004, 15, 3131. (b) Borah, J.C.; Gogoi, S.; Boruwa, J.; Kalita, B.; Nabin C. Barua, N.C. Tetrahedron Lett. 2004, 45, 3689. (c) Castagnolo, D.; Armaroli, S.; Corelli, F.; Botta, M. Tetrahedron: Asymm. 2004, 15, 941. (d) . Voronkov, M.V.; Gontcharov, A.V.; Wang, Z.M. Tetrahedron Lett. 2003, 44, 407. (e) Hamamoto, H.; Mamedov, V.A.; Kitamoto, M.; Hayashi, N.; Tsuboi, S. Tetrahedron: Asymm. 2000, 11, 4485. (f) Wuts, P.G.M.; Gu, R.L.; Northuis, J.M. Tetrahedron: Asymm. 2000, 11, 2117. (g) Ha, H.J.; Park, G.S.; Ahn, Y.G.; Lee, G.S. Bioorg. Med. Chem. Lett. 1998, 8, 1619. (h) Lee, K.Y.; Kim, Y.H.; Park, M.S.; Ham, W.H. Tetrahedron Lett. 1998, 39, 8129. (i) Adger, B.M.; Barkley, J.V.; Bergeron, S.; Cappi, M.W.; Flowerdew, B.E.; Jackson, M.P.; McCague, R.; Nugent, T.C.; Roberts, S.M. J. Chem. Soc., Perkin Trans. 1, 1997, 3501.

6. (a) Katsuki, T.; Sharpless, K. B. J. Am. Chem. Soc. 1980, 102, 5974. (b) Jacobsen, E. N.; Makro, A. I.; Mungall, W. S.; Schroder, G.; Sharpless, K. B. J. Am. Chem. Soc. 1988, 110, 1968. (c) Li, G.; Chang, H. T.; Sharpless, K. B. Angew. Chem., Int. Ed. Engl. 1996, 35, 451.

7. (a) Arai, S.; Ishida,T.; Shioiri, T. Tetrahedron Lett. 1998, 39, 8299. (b) Wang, Y.C.; Li, C.L.; Tseng, H.L.; Chuang, S.C.; Yan, T.H. Tetrahedron: Asymm. 1999, 10, 3249. (c) Arai, S.; Suzuki, Y.; Tokumaru, K.; Shioiri, T. Tetrahedron Lett. 2002, 43, 833. (d) Mamaghani, M.; Khalil Tabatabaeian, K.; Ghanadzadeh, A.; Habibi, F. Tetrahedron Lett. 2003, 44, 4775. (e) Arai, S.; Tokumaru, K.; Aoyama, T. Tetrahedron Lett. 2004, 45, 1845. (f) Commercon, A.; Bezard, D.; Bernard, F.; Bouzart, J.D. Tetrahedron Lett. 1992, 33, 5185.

8. Coşkun, N.; Er, M. Unpublished

9. For a review and recent examples of azide reductions see: (a) Scriven, E. F. V.; Turnbull, K. Chem. Rev. 1988, 88, 297. (b) Zimmermann, V.; Avemaria, F.; Brase, S. J. Comb. Chem, 2007, 9, 200. (c) Sridhar, P.R.; Prabhu, K.R.; Chandrasekaran, S. J. Org. Chem. 2003, 68, 5261. (d) Lee, J.W.; Fuchs, P.L. Org. Lett. 1999, 1, 179. (e) Batanero, B.; Escudero, J.; Barba, F. Org. Lett. 1999, 1, 1521. (f) Peters, R.G.; Warner, B.P.; Burns, C.J. J. Am. Chem. Soc. 1999, 121, 5585. (g) Hays, D.S.; Fu, G.C. J. Org. Chem. 1998, 63, 2796. (h) Benati, L.; Bencivenni, G.; Leardini, R.; Minozzi, M.; Nanni, D.; Scialpi, R.; Spagnolo, P.; Zanardi, G. J. Org. Chem. 2006, 71, 434. (i) Vankar, Y.D.; Kumaravel, G.; Mukherjee,N. and Rao, C.T. Synth. Commun. 1987, 17, 181. (j) Vankar, Y.D.; Chaudhuri, N.C. and Rao, C.T. Tetrahedron Lett. 1987, $28,551$.

10. Srivastava, R. P.; Zjawiony, J. K.; Peterson, J. R.; McChesney, J. D. Tetrahedron: Asymm. 1994, 5, 1683. 